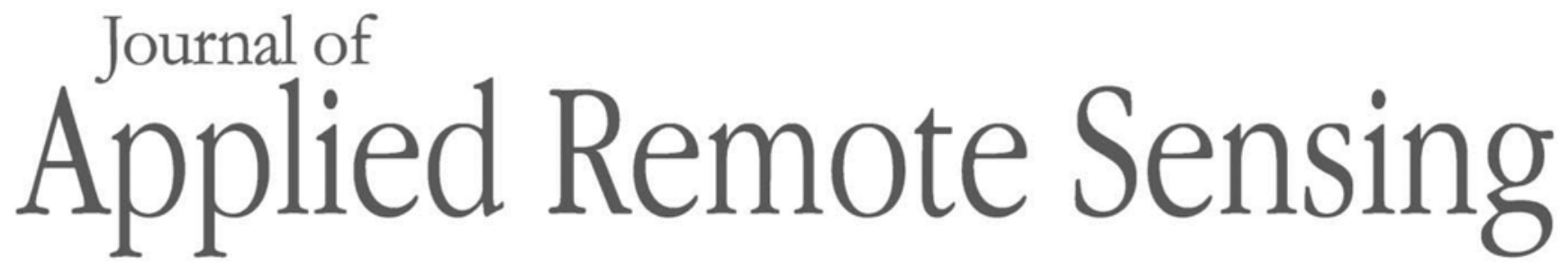

RemoteSensing.SPIEDigitalLibrary.org

\title{
GOES-16 Advanced Baseline Imager visible near-infrared channel low- light signal-to-noise ratio
}

Robbie Iacovazzi

Xiangqian $\mathrm{Wu}$ 


\title{
GOES-16 Advanced Baseline Imager visible near-infrared channel low-light signal-to-noise ratio
}

\author{
Robbie Iacovazzi ${ }^{\mathrm{a}, *}$ and Xiangqian $\mathrm{Wu}^{\mathrm{b}}$ \\ ${ }^{\mathrm{a}} \mathrm{GST}$, Inc., Greenbelt, Maryland, United States \\ ${ }^{b}$ NOAA/NESDIS/STAR, College Park, Maryland, United States
}

\begin{abstract}
Quantifying Geostationary Operational Environmental Satellite R-series (GOES-R) Advanced Baseline Imager (ABI) Channel (Ch) 2 low-light signal-to-noise ratio (SNR) is a core $\mathrm{ABI}$ postlaunch product test to validate prelaunch performance expectations of this parameter. For GOES-16, an SNR analysis has been performed to accomplish this goal using ABI Mode-3 MESO visible near-infrared (VNIR) channel L1b radiance product images taken every $30 \mathrm{~s}$ between 17:00 UTC and 17:15 UTC on May 23, 2017. Scene pixel data used in the analysis are first screened to ensure they meet the low-light criteria and to minimize scene temporal variability associated with meteorological evolution and geolocation uncertainty in highly inhomogeneous scenes. After such screening, image-to-image radiance time difference statistics are compiled and analyzed to estimate instrument SNR. Based on GOES-R ABI vendor prelaunch testing, minimum and mean expected ABI Ch 2 postlaunch SNR performance at 5\% albedo was estimated to be 44.2 and 64.5 , respectively. Meanwhile, the SNR computed in this analysis is found to be about $57 \pm 8$. Thus, low-light SNR for ABI Ch 2 is shown to meet minimum user expectations. The analysis for the other five GOES-16 ABI VNIR channels is also shown to contrast the SNR for these channels. (C) The Authors. Published by SPIE under a Creative Commons Attribution 4.0 Unported License. Distribution or reproduction of this work in whole or in part requires full attribution of the original publication, including its DOI. [DOI: 10.1117/1.JRS.14.026502]
\end{abstract}

Keywords: GOES-R; Advanced Baseline Imager; visible near-infrared; radiance, calibration, validation, signal-to-noise ratio.

Paper 190767 received Oct. 1, 2019; accepted for publication Mar. 19, 2020; published online Apr. 6, 2020.

\section{Introduction}

The Geostationary Operational Environmental Satellite R-series (GOES-R) Mission Requirements Document (MRD $;^{1}$ GOES-R Series Program) captures National Oceanic and Atmospheric Administration's (NOAA's) consolidated L1b and L2+ meteorological satellite product suite accuracy, precision, and geographical coverage needs. These MRD product requirements in turn determine MRD instrument requirements. For example, in the GOES-R Advanced Baseline Imager (ABI) Suspended Matter/and Aerosol Algorithm Theoretical Basis Document, ${ }^{2}$ the ABI aerosol optical depth (AOD) L2+ product performance has been shown to have sensitivity to the ABI $0.64 \mu \mathrm{m}$ band [Channel (Ch) 2] signal-to-noise ratio (SNR) in conditions where surface albedo is small-e.g., about 5\%. This sensitivity in turn has driven the following ABI Ch 2 low-light SNR MRD requirement: "The GOES-R Space Segment shall produce Radiance product observations in low light (5\% albedo) conditions in the $0.64 \mu \mathrm{m}$ band at a 50:1 SNR." It was determined that this specification was too strict during the course of ABI prelaunch testing, and was relaxed to 20:1 after being deemed acceptable by product teams and stakeholders.

After the launch of each GOES-R ABI instrument, it is essential to validate the performance of all products. During GOES-R development and acquisition, a suite of ABI postlaunch product test (PLPT) activities was defined for the purpose of postlaunch product validation and assessment. In a nutshell, each PLPT activity is defined by an overarching product or instrument specification that motivates the test, along with an associated test objective, method description, and success criteria. In addition, each PLPT description outlines the required instrument

*Address all correspondence to Robbie Iacovazzi, E-mail: Robert. Iacovazzi@noaa.gov 
configuration and operation needed to carry out the test, as well as the test prerequisites and data capture requirements. All PLPT activities are carefully defined in a suite of GOES-R ABI Beta, Provisional and Full Validation Readiness, Implementation and Management Plan documents for all ABI products. ${ }^{3}$

Once a PLPT is executed, the resultant test data are analyzed, and test success is determined by comparing the results to prelaunch formulated baseline values of ABI expected postlaunch performance. GOES-R subject-matter experts, using the data from prelaunch bench and thermal vacuum $A B I$ instrument testing performed by the $A B I$ vendor, established the expected postlaunch performance baseline of each $\mathrm{ABI}$ product and many associated instrument parameters.

This article focuses on one PLPT activity, ABI-L1b-PLPT-009, which validates ABI visible near-infrared (VNIR) channel low-light SNR. In particular, the primary test goal is to validate ABI Ch 2 low-light SNR with respect to its baseline performance minimum and mean expected values. For GOES-16, these minimum and mean values are 44.2 and 64.5, respectively. After launch of GOES-16 on November 19, 2016, there was one opportunity during its postlaunch test period to perform this PLPT. This paper offers results from the GOES-16 ABI implementation of this test. Section 2 of this article is an overview of the test method. Section 3 provides results from the test, and Sec. 4 offers a brief summary of the work.

\section{Advanced Baseline Imager Visible Near-Infrared Channel Low-Light Signal-to-Noise Ratio Monitoring and Analysis Method}

The foundation for this test is repeated collocated image acquisition by ABI of an Earth region of interest with a temporal resolution as fine as possible to eliminate time-related scene evolution. The ABI instrument has two timelines, Mode 3 and Mode 6, which capture the mesoscale images with a frequency of $30 \mathrm{~s} .{ }^{4,5}$ Each mesoscale region is about $1000 \times 1000 \mathrm{~km}$ and 30 (20) images are taken over the course of the $15 \mathrm{~min}(10 \mathrm{~min})$ in Mode-3 (Mode-6) timeline. Within each timeline, it is possible to set two image locations, called MESO-1 and MESO-2, which can alternate imagery between two Earth scenes. This degrades the temporal frequency to $1 \mathrm{~min}$ for each mesoscale scene. For this test, MESO-1 and MESO-2 are fixed to the same location, to take advantage of ABI's higher 30-s image cadence. The test is performed only once during PLPT, after the ABI L1b product reaches beta-validated status. Beta status is characterized by an end state where the product has been minimally validated and may still contain significant errors. So, it is most important to show that this method can perform during the instrument's postlaunch tests, even when the product quality is not well understood and not optimal.

For collocated ABI MESO-1 and MESO-2 image pair centers, every grid point in the image is viewed once every $30 \mathrm{~s}$ within a 15 -minute MODE- 3 timeline. This gives 30 collocated images per timeline. Assuming that scene change between 30-s images is negligible, and that image navigation and registration (INR) error is also very small, at-sensor radiance would be almost identical for consecutive mesoscale images. Therefore, sensor-measured radiance differences for the same pixel for each mesoscale image pair would be purely due to instrument noise. The standard deviation of pixel temporal radiance differences for all pixels with similar radiance accumulated over the 15-min ABI Mode-3 timeline from all 29 ABI MESO image pairs is the radiometric noise at that level of signal. SNR can be calculated for a given population within a prescribed radiance range as the square root of two times the ratio of the temporal mean radiance to the standard deviation of image-to-image temporal radiance differences. In this report, this is called "Temporal SNR," which can also be expressed as follows.

Temporal SNR $\left(\mathbf{S N R}_{T}\right)$ : For all scene pixels, $n=1$ to $N$, that pass through radiance and spatial SNR (defined later) filtering threshold values for consecutive images at time $t$ and $t+1$,

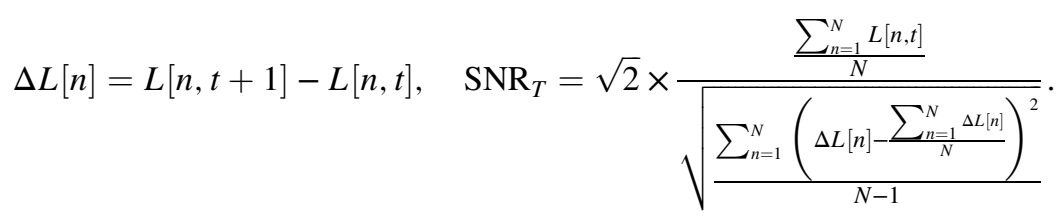

In the above equation, $L[n, t+1]$ and $L[n, t]$ denotes the radiance for a single pixel at times $t+1$ and $t$, respectively. Meanwhile, $\Delta L[n]$ is the radiance difference for pixel $n$. The parameter $N$ is 
the population of $\Delta L[n]$ values accumulated from all 29 ABI MESO image pairs. Note that typically SNR is determined from a single image over a uniform scene, and not the difference between scenes. For this reason, a factor of $\sqrt{2}$ is applied to the $\mathrm{SNR}_{T}$ equation due to the fact that the difference of two independent populations can increase the standard deviation by this factor. This becomes a way to estimate SNR at various signal intensities, in particular at the apparent reflectance of $5 \%$ for band 2 as required by MRD.

It is important to note that the ABI L1b radiance product has discrete values, and so there may be many instances where $\Delta L[n]$ is exactly zero. This is a consequence of the limited bit depth (10 to 12 bits) of the ABI L1b product data compared to the full bit depth (14 bits) of the raw L0 data. In the extreme, small instrument radiometric noise compared to quantized radiance difference can lead to highly overestimated SNR. To address this issue, a more conservative estimate of SNR is evaluated by replacing the all occurrences of $\Delta L[n]=0$ with root-two times the $\mathrm{L} 1 \mathrm{~b}$ radiance scaling factor and a random sign. The parameter that is created by this more conservative method of computing temporal SNR is called adjusted temporal SNR or SNR $_{T \text { adj. }}$.

In the nominal ABI 30-s mesoscale image cadence, the assumptions proposed earlier of a negligibly changing scene and nearly perfect INR are superseded by the need to consider possible scene changes due to meteorological and solar illumination evolution and INR uncertainties in an inhomogeneous scene. Meteorological evolution could include changes in cloudiness or aerosols, while solar illumination changes are due to the diurnal cycle. In this study, meteorological evolution in a scene and/or geolocation uncertainty in an inhomogeneous scene-e.g., with cloud or clear sky coastline edges-are considered to be potential significant factors in creating temporal radiance variability between 30-s MESO images. The effect of the solar diurnal cycle is considered to be negligible. In addition, the quantization of the radiance data product restricts the methods ability to resolve SNR.

As previously mentioned, most ABI channels are characterized as scaled integers with a bit depth of 12 or smaller. So, "bit-depth error" can be a limiting factor in the analysis that needs to be quantified. This can be expressed as follows.

Quantization SNR: The maximum SNR resolvable at a given radiance level as a result of L1b product quantization into scaled integers before being packaged into the GOES Rebroadcast data stream. In this paper, this is expressed as

$$
\mathrm{SNR}_{Q}=\sqrt{2} \times \frac{L}{S_{s i}}
$$

where $L$ is the scaled radiance and $S_{s i}$ is the ABI L1b radiance product scaled integer slope reported in the GOES-R L1b Product Description and Users' Guide. ${ }^{6}$

So, radiance product discretization is used not only to adjust the temporal SNR to account for its uncertainties but also to define the SNR resolution limit where the only errors are due to the limited bit depth.

To filter mesoscale scene regions that may be subject to temporal radiance changes arising from meteorological evolution or INR instability in highly inhomogeneous scenes, a spatial SNR is computed for each scene pixel. For each $3 \times 3$ group of pixels, the standard deviation of the pixel group is determined.

Spatial SNR: The ratio of the radiance of the center pixel of each $3 \times 3$ group to the standard deviation of the pixel group and can be expressed as

$$
\mathrm{SNR}_{\text {Spatial }}=\frac{L[5, t]}{\sqrt{\frac{\sum_{m=1}^{9}\left(L[m, t]-\frac{\sum_{m=1}^{9} L[m, t]}{9}\right)^{2}}{8}}},
$$

where $L[m, t]$ is the radiance at a given ABI fixed grid $3 \times 3$ subpixel region depicted in Fig. 1 . Note that when all nine pixels have the same radiance, then spatial SNR, SNR $_{\text {spatial }}$, has an unrealistic value of infinity. To mitigate this, the maximum resolvable SNR, defined by the quantization $\mathrm{SNR}\left(\mathrm{SNR}_{Q}\right)$ parameter, is substituted. Also, pixel subregions where all pixels do not have valid data are not considered in processing. 


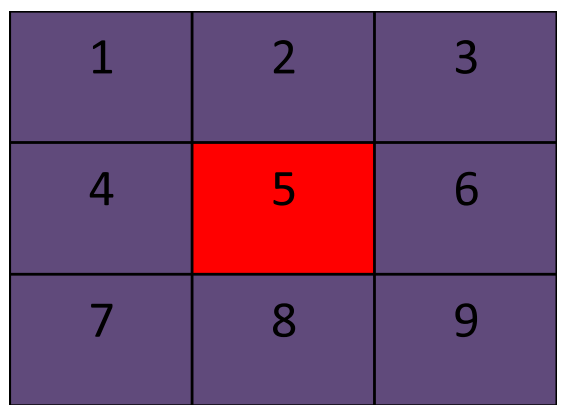

Fig. 1 An illustration of an $A B I$ fixed grid $3 \times 3$ subpixel region.

Pixels from each ABI mesoscale image are eliminated from processing when they have relatively small $\mathrm{SNR}_{\text {spatial, }}$, which usually is associated with cloud edges, coastlines, desert boundaries, etc. The $\mathrm{SNR}_{\text {spatial }}$ threshold is determined by trying to find a reasonable balance between adequate sample size and achieving a minimum temporal radiance difference variability that is not artificially skewed. How this is done is discussed in Sec. 3 of the paper. When processing a given pixel, if consecutive images, or only one mesoscale image, have an $\mathrm{SNR}_{\text {spatial }}$ that is below the prescribed threshold, that pixel is not considered in the analysis. In addition, fill or zero value data are eliminated, as well as regions where the ABI L1b Data Quality Flag is nonzero. Computing $\mathrm{SNR}_{T}$ from the remaining data then gives an overall estimate of SNR for the L1b product. It is important to note here that the ABI L1b radiance product is composed of fixed grid pixel values. These values are computed from detector sample observations that are remapped to the fixed grid using a spatial convolution kernel. After the remapping process, the detector samples used to determine the radiance value at a given pixel cannot be determined. Therefore, this method is not capable of estimating the SNR for individual ABI detectors.

\section{GOES-16 ABI-L1b-PLPT-009 Low-Light Signal-to-Noise Ratio Results}

In this section, GOES-16 ABI VNIR channel SNR results are given for a PLPT MESO-1 and MESO-2 data acquisition centered at the Earth coordinates $8.975615^{\circ} \mathrm{S}$ and $76.91671^{\circ} \mathrm{W}$, and taken between 17:00 and 17:15 UTC on May 23, 2017. This site, depicted in Fig. 2, was chosen because it contains water bodies and desert, dense vegetation, elevation between sea level and $5.52 \mathrm{~km}$, and a variety of reflectance. The plethora of regions of interest in this scene can be equated to the ABI VNIR channels in the following way: clear land at higher elevation for the $0.47-\mu \mathrm{m}$ band (Ch 1); clear and relatively dark ocean pixels for the $0.64-\mu \mathrm{m}$ band (Ch 2 ); clear ocean pixels for the $0.86-\mu \mathrm{m}$ band (Ch 3) and $1.61-\mu \mathrm{m}$ band (Ch 5); higher altitude clouds for the 1.378- $\mu \mathrm{m}$ band (Ch 4); and clear dark land pixels for the 2.26- $\mu \mathrm{m}$ band (Ch 6).

The results quantify region of interest values $\mathrm{SNR}_{T}, \mathrm{SNR}_{T \mathrm{adj}}$, average $\mathrm{SNR}_{\text {spatial }}$, and $\mathrm{SNR}_{Q}$ for five radiance subintervals between $2.5 \%$ and $7.5 \%$ albedo, where each radiance subinterval is expressed as

$$
\begin{gathered}
L_{L}=\{0.025+0.01 \times(S I-1)\} \cdot \frac{E_{\mathrm{sun}} \cos (0) d_{r}}{\pi}, \\
L_{H}=\{0.025+0.01 \times(S I)\} \cdot \frac{E_{\mathrm{sun}} \cos (0) d_{r}}{\pi}, \\
d_{r}=1 .
\end{gathered}
$$

In these equations, $L_{L}$ and $L_{H}$ are estimate of low and high radiance bounds of the $S I$ 'th radiance range subinterval, where $S I$ is a number between 1 and 5 . The parameter $E_{\text {sun }}$ is the in-band total solar irradiance acquired from the ABI L1b metadata, and $d_{r}$ is the square of the inverse EarthSun distance in astronomical units. The radiance subintervals established here are approximated using a solar zenith angle (SZA) of zero degrees, which establishes back-of-the-envelope 


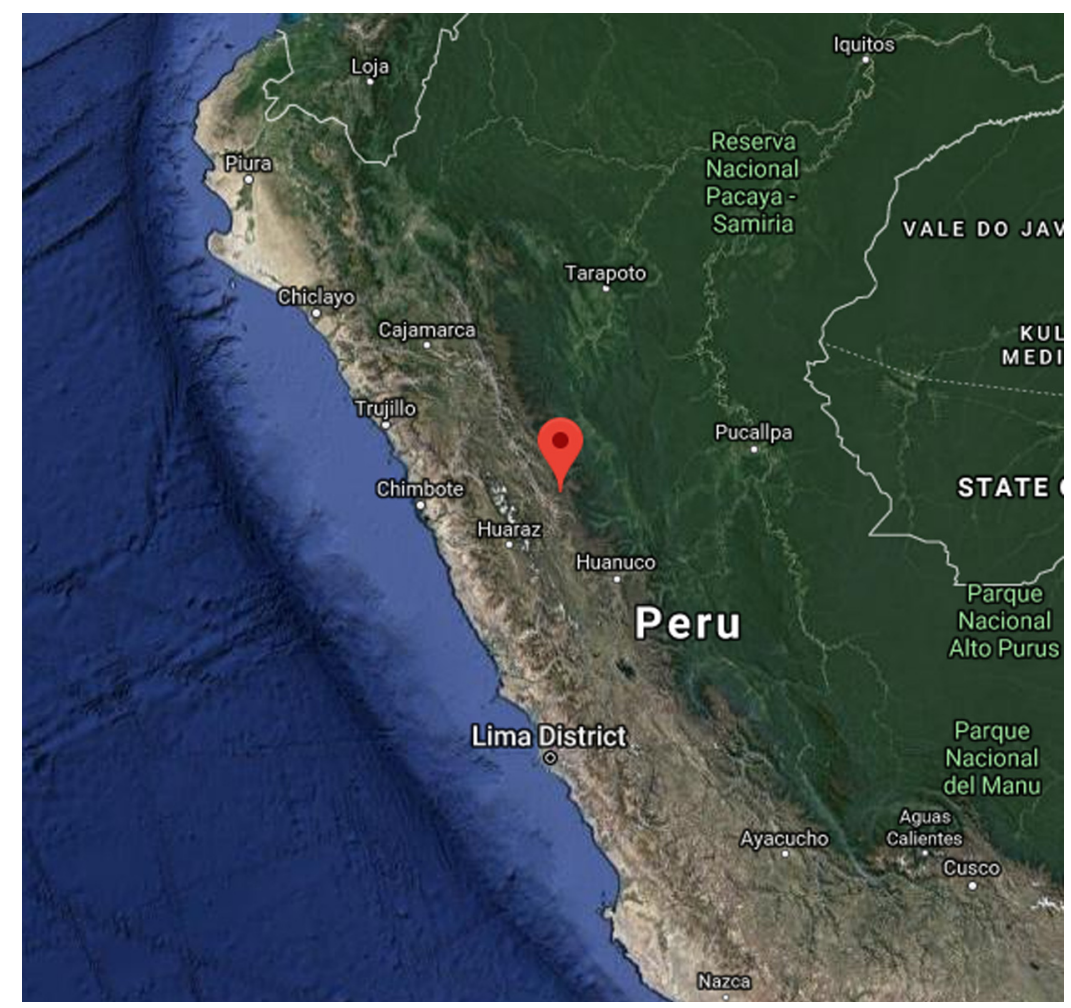

Fig. 2 ABI MESO site used for this analysis. Courtesy: Google Maps.

radiance intervals for a given albedo range. The full albedo range from $2.5 \%$ to $7.5 \%$ should establish a radiance range that includes actual scene radiances from a 5\% albedo scene, even if the SZA is off by 25 to $35 \mathrm{deg}$. Doing the analysis in this way ensures that scenes with radiances that also contain 5\% albedo scenes are captured, which is the albedo at which the GOES-R ABI Ch 2 low-light SNR requirement is written. Also, the use of five subintervals between $2.5 \%$ and $7.5 \%$ restricts albedo to within about $0.5 \%$ for the population of scenes that have near $5 \%$ albedo. In the following sections, detailed results will be presented for GOES-16 ABI Ch $2(0.64 \mu \mathrm{m})$, while less-detailed, salient results will be given for the other VNIR channels $-0.47 \mu \mathrm{m}$ (Ch 1), $0.86 \mu \mathrm{m}$ (Ch 3), $1.38 \mu \mathrm{m}$ (Ch 4), $1.61 \mu \mathrm{m}$ (Ch 5), and $2.26 \mu \mathrm{m}$ (Ch 6).

\subsection{Advanced Baseline Imager Channel 2 Low-Light Signal-to-Noise Ratio}

For Ch 2 , the full radiance range associated with low albedo range is $13.0 \mathrm{~W} /\left(\mathrm{m}^{2} \mathrm{sr} \mu \mathrm{m}\right)$ to $38.9 \mathrm{~W} /\left(\mathrm{m}^{2} \mathrm{sr} \mu \mathrm{m}\right)$. The $\mathrm{SNR}_{\text {spatial }}$ threshold parameter-needed to filter out temporal radiance changes arising from meteorological evolution, or INR instability coupled with large scene inhomogeneity-has a threshold that is set to 39.4. The manner in which this threshold is determined is discussed later in this section and in Appendix A. A two-dimensional histogram of $\Delta L[n]$ versus $\mathrm{SNR}_{\text {spatial }}$, for the population of samples accumulated over the 15-min ABI Mode-3 timeline from all 29 ABI MESO image pairs within the radiance range, is shown in Fig. 3. In this figure, the temporal radiance difference can be as high as $+13 \mathrm{~W} /\left(\mathrm{m}^{2} \mathrm{sr} \mu \mathrm{m}\right)$ and as low as $-16 \mathrm{~W} /\left(\mathrm{m}^{2} \mathrm{sr} \mu \mathrm{m}\right)$. For those points that exceed the $\mathrm{SNR}_{\text {spatial }}$ threshold range, the $\Delta L[n]$ variability decreases by at least $80 \%$ to between $\pm 3 \mathrm{~W} /\left(\mathrm{m}^{2} \mathrm{sr} \mu \mathrm{m}\right)$. In Fig. 4, a map of all 17:00 UTC May 23, 2017 ABI Ch 2 L1b radiance values that pass the SNR $_{\text {spatial }}$ threshold is shown. This figure shows that a predominance of low radiance values lie over ocean, many of which are spatially contiguous. If there are any low radiance scenes over land, they have relatively small $\mathrm{SNR}_{\text {spatial }}$ and thus are screened out. Finally, some mild striping artifacts also surface, which is not uncommon for preoperational satellite data.

The histograms in Fig. 5 reveal the distribution of temporal radiance difference for each of the 29 temporally contiguous ABI Ch 2 mesoscale image pairs in this Mode- 3 timeline for pixels 


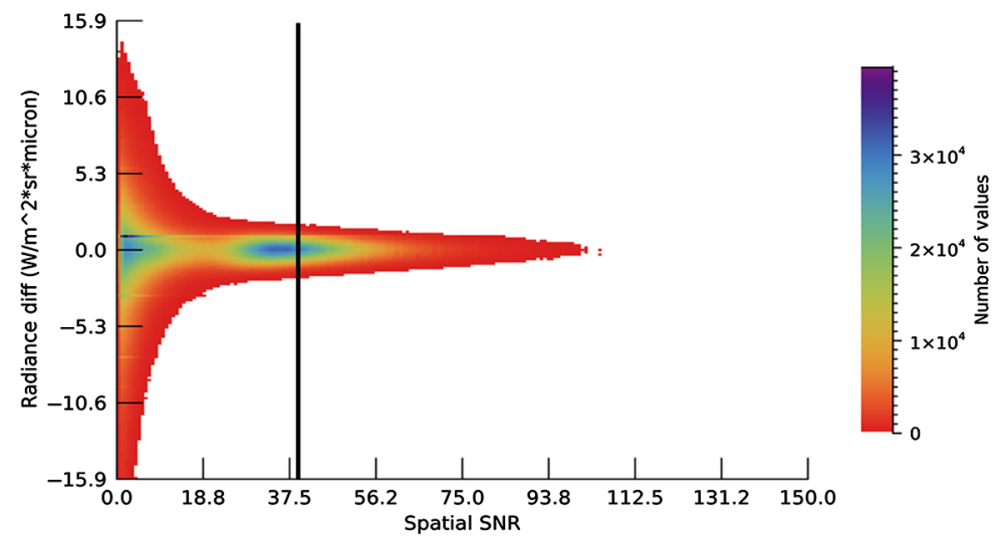

Fig. $3 \mathrm{ABI} C h 2$ two-dimensional histogram of temporal radiance difference $(\Delta L[n])$ versus spatial SNR $\left(S_{\text {spatial }}\right)$ for all 29 ABI MESO radiance scene pairs between 17:00 and 17:15 UTC on May 23, 2017. The black line is the $\mathrm{SNR}_{\text {spatial }}$ threshold.

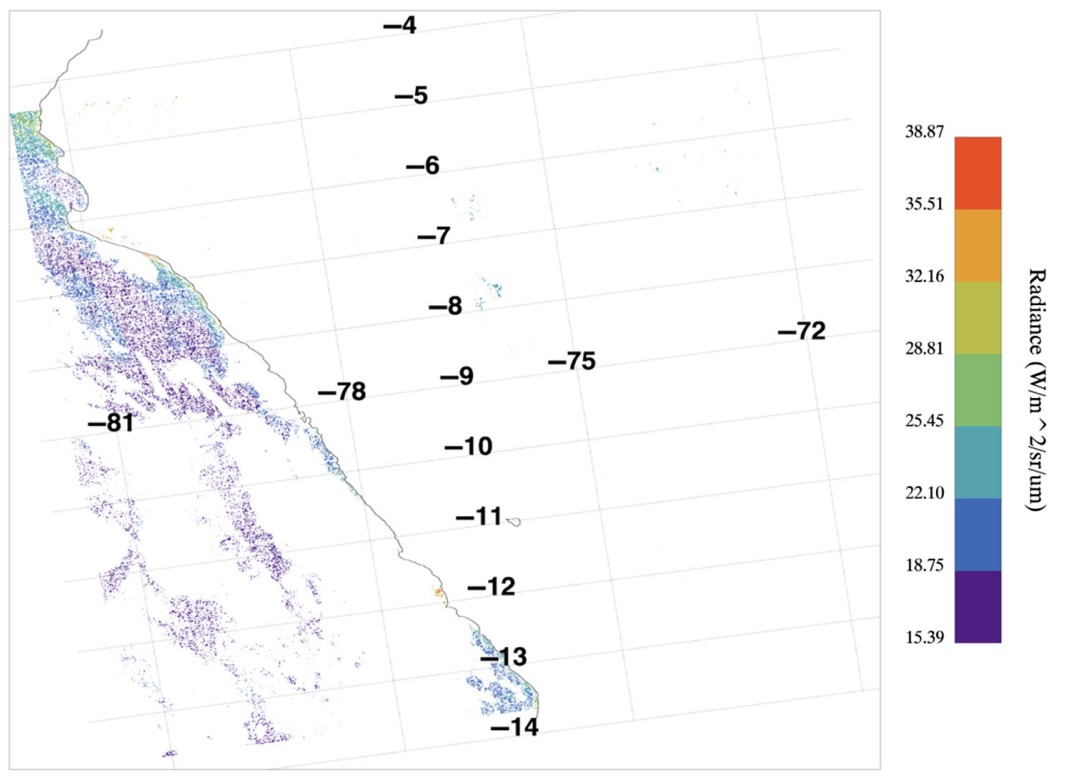

Fig. 4 A map of $A B I$ Mode-3 Ch 2 L1b radiance for 17:00 UTC on May 23, 2017 for those pixels that exceed the $\mathrm{SNR}_{\text {spatial }}$ threshold.

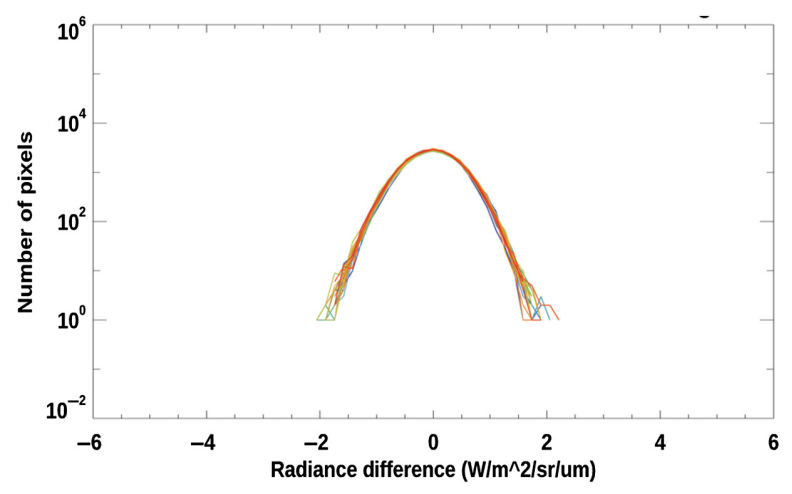

Fig. $5 \mathrm{ABI}$ Mode-3 Ch $2 \mathrm{~L} 1 \mathrm{~b}$ radiance differences histograms for the 29 consecutive MESO image pairs taken between 17:00 and 17:15 UTC on May 23, 2017. The acquisition time is represented by line color, where red (purple) is the beginning (end) of the Mode- 3 timeline. The data are within the low-albedo range and pass through the $\mathrm{SNR}_{\text {spatial }}$ threshold. 


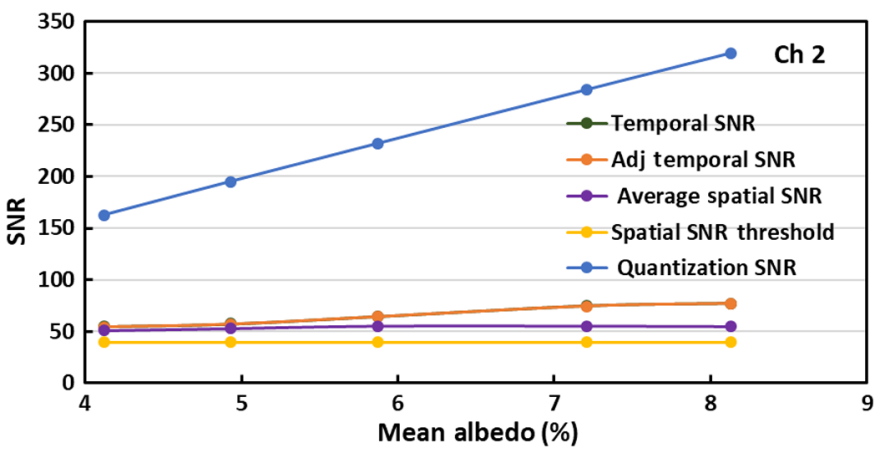

Fig. 6 For $\mathrm{ABI} C h$ 2, the following parameters are depicted as a function of mean albedo: average and threshold of $\mathrm{SNR}_{\text {spatial }}$; $\mathrm{SNR}_{Q}$; and $\mathrm{SNR}_{T}$ computed with and without substituting zero values of temporal radiance difference with the quantization radiance difference.

that are within the radiance range and exceed the $\mathrm{SNR}_{\text {spatial }}$ threshold. Analysis from each of the contiguous mesoscale image pairs shows that almost all values lie between $\pm 3 \mathrm{~W} /\left(\mathrm{m}^{2} \mathrm{sr} \mu \mathrm{m}\right)$. It also shows a relatively large peak number of samples $\left(>10^{3}\right)$ and exhibits very little variation between the 29 plots, except near the wings of the distribution.

As shown in Fig. 6, a plot of SNR versus mean actual albedo shows that data are present from all five radiance subranges, which have actual mean albedo values of $4.1 \%, 4.9 \%, 5.9 \%, 7.2 \%$, and $8.1 \%$. Note that the actual mean albedo values do not fall within the five ranges- $2.5 \%$ to $3.5 \%, 3.5 \%$ to $4.5 \%, 4.5 \%$ to $5.5 \%, 5.5 \%$ to $6.5 \%$, and $6.5 \%$ to $7.5 \%$-initially defined to determine the radiance intervals used to acquire the ABI radiance data. The GOES-R ABI radiance product does not report a surface albedo value, so to gather the ABI MESO scene radiance pixel data that are near $5 \%$ albedo, a back-of-the-envelope value of overall scene in-band solar irradiance (ABI Actual), Earth-Sun distance ( $1 \mathrm{AU})$, and SZA ( $0 \mathrm{deg})$ was used to establish approximate radiance intervals between $2.5 \%$ and $7.5 \%$. When actual ABI radiance measurements are detected within these radiance intervals, the surface albedo is computed from them using actual ABI in-band solar irradiance, Earth-Sun distance, and SZA reported in the GOES-R ABI L1b radiance product metadata. ${ }^{6}$ Actual albedo computed from ABI radiances can thus be higher than those used to help screen the radiance data. This data-gathering approach is adequate to find a sufficient population of data in the scene that have albedo near our $5 \%$ target, and it saves us from computing albedo for the entire ABI MESO images before screening.

The average $\mathrm{SNR}_{\text {spatial }}$ in Fig. 6 varies between 51 and 55, while $\mathrm{SNR}_{Q}$ monotonically increases from 164 to 318 as mean actual albedo increases from $4.1 \%$ to $8.1 \%$. This reflects that the quantization of the product radiance as a result of the limited 12-bit bit depth is constant, while the radiance itself increases monotonically. The average values of $\mathrm{SNR}_{T}$ associated with the five albedo values are 55, 57, 65, 75, and 77. The values of adjusted temporal SNR, or $\mathrm{SNR}_{T \text { adj }}$, are only slightly lower- $54,57,64,74$, and 77 . The reason for this only slight difference between $\mathrm{SNR}_{T}$ and $\mathrm{SNR}_{T \text { adj }}$ is due to the relatively large quantization $\mathrm{SNR}_{Q}$ values for this channel, which is reported as a 12-bit scaled integer.

As mentioned in Sec. 1, the GOES-R MRD ${ }^{1}$ defines low-light SNR as the SNR for a scene in low-light (5\% albedo) conditions. Subject-matter experts within GOES-R Program Systems Engineering established a prelaunch estimate of expected (baseline) postlaunch minimum and mean performance of ABI Ch 2 low-light SNR of 44.2 and 64.5, respectively. This analysis shows that, based on the imposed values of $\mathrm{SNR}_{\text {spatial }}$ threshold, this expected value is validated, as $\mathrm{SNR}_{T}$ of 57 is between the minimum and mean values. But how conclusive is this $\mathrm{SNR}_{T}$ estimate?

Figure 3, shown earlier, reveals ABI Ch 2 pixel radiance difference between images in time versus $\mathrm{SNR}_{\text {spatial }}$. This plot is an accumulation of all pixel pairs with scene albedo between $2.5 \%$ and $7.5 \%$ for all 29 image pairs in the 15-min ABI timeline. This figure shows that as $\mathrm{SNR}_{\text {spatial }}$ increases, which reflects increased scene homogeneity, the pixel radiance difference between consecutive images has a tightened range of values around zero. The SNR spatial $_{\text {threshold of } 39.4}$ shown earlier was chosen to ensure adequate sampling and to screen out high scene variability 
artifacts that can eclipse our ability to estimate low-light SNR. Further details justifying the choice of $\mathrm{SNR}_{\text {spatial }}$ threshold are given in the following.

In Fig. 7, the parameters $\mathrm{SNR}_{T}, \mathrm{SNR}_{T \text { adj }}$, and population sample count are plotted as a function of $\mathrm{SNR}_{\text {spatial }}$ threshold. Only pixels with $\mathrm{SNR}_{\text {spatial }}$ greater than $\mathrm{SNR}_{\text {spatial }}$ threshold in consecutive images are used to estimate $\mathrm{SNR}_{T}$ and $\mathrm{SNR}_{\text {Tadj. }}$. This figure shows that as $\mathrm{SNR}_{\text {spatial }}$ threshold increases from about 0 to 80, the measure of $\mathrm{SNR}_{T}$ and $\mathrm{SNR}_{T \text { adj }}$ for $\mathrm{Ch} 2$ increases from about 16 to 90 while the analysis pixel count in the analysis drops from about $2 \times 10^{6}$ to about $1 \times 10^{3}$. In addition, because of the relatively refined 12-bit bit depth, $\mathrm{SNR}_{T}$ and $\mathrm{SNR}_{\text {Tadj }}$ are very similar, as they are not seen as significantly separated in the figure.

To unequivocally interpret these results, we would need to be able to separate the temporal radiance changes due to instrument noise from those caused by scene radiance variations manifested into temporal variations. As mentioned in Sec. 2, temporal radiance variability between ABI 30-s mesoscale image acquisitions can mainly be caused by meteorological evolution within the scene, as well as geolocation uncertainty when viewing an inhomogeneous scene. Since a conclusive method to decompose the individual contributions to temporal scene variability has not been determined, we are forced to make some assumptions in approaching our analysis.

The first assumption is that instrument noise for a given radiance signal is the same regardless of the magnitude of scene spatial variability. The second is that improving our ability, or confidence, to discern $\mathrm{SNR}_{T}$ due to instrument noise requires an understanding of how implementing an $\mathrm{SNR}_{\text {spatial }}$ threshold affects both average $\mathrm{SNR}_{\text {spatial }}$ and $\mathrm{SNR}_{T}$. In Fig. 7, $\mathrm{SNR}_{T}$ continues to increase to near 90 as $\mathrm{SNR}_{\text {spatial }}$ threshold increases, but the prelaunch expected mean value of $\mathrm{SNR}_{T}$ is 64.5 . Insight into this questionable result is aided by Fig. 8, which shows change of $\mathrm{SNR}_{T}$ with respect to change of average $\mathrm{SNR}_{\text {spatial }}\left(\mathrm{dSNR}_{T} / \mathrm{dSNR}_{\text {spatial }}\right)$ as a function of $\mathrm{SNR}_{\text {spatial }}$ threshold. Figure 8 reveals three $\mathrm{dSNR}_{T} / \mathrm{dSNR}_{\text {spatial }}$ regimes, which are characterized, respectively, by an initial steep decline followed by two lower plateaus. The first plateau has a value $\mathrm{dSNR}_{T} / \mathrm{dSNR}_{\text {spatial }}$ of 0.9 , while the second plateau has an average value of about 0.75 . These are explained as follows, and a more theoretical treatment is offered in Appendix A.

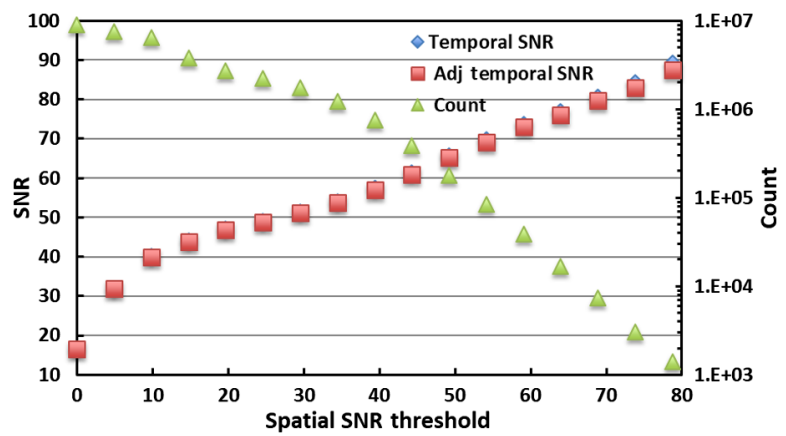

Fig. 7 The $\mathrm{SNR}_{T}, \mathrm{SNR}_{T \text { adj }}$, and population sample count as a function of $\mathrm{SNR}_{\text {spatial }}$ threshold.

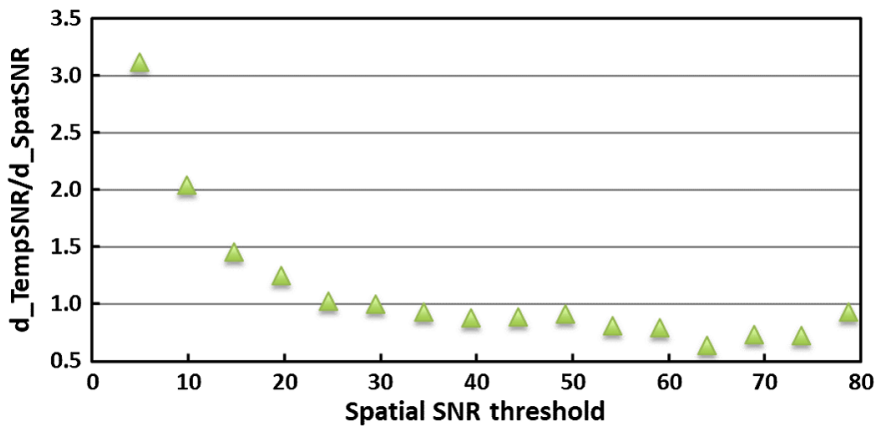

Fig. 8 The parameter $d S N R_{T} / d S N R_{\text {spatial }}$ as a function of $S N R_{\text {spatial }}$ threshold. 
The first regime contains low $\mathrm{SNR}_{\text {spatial }}$ scenes with high spatial variability that may include high-contrast dynamic meteorological features, such as clouds. Such scenes can easily induce temporal variability and corresponding low $\mathrm{SNR}_{T}$ values. In this case, instrument noise represents a relatively small contribution to $\mathrm{SNR}_{T}$. So, as $\mathrm{SNR}_{\text {spatial }}$ threshold increases for low $\mathrm{SNR}_{\text {spatial }}$ scenes, these highly variable scenes are screened out, which eliminates pixels with a great deal of temporal variability as well. Thus, in this case, $\mathrm{SNR}_{T}$ increases more rapidly than average $\mathrm{SNR}_{\text {spatial }}$ when $\mathrm{SNR}_{\text {spatial }}$ threshold is increased. The parameter $\mathrm{dSNR}_{T} / \mathrm{dSNR}_{\text {spatial }}$ will be $>1$ in this regime but will decrease as SNR $_{\text {spatial }}$ threshold increases, as the "worst offender" spatial variability pixels become less predominant in the analysis. In Fig. 8, this regime extends from an $\mathrm{SNR}_{\text {spatial }}$ threshold of 0 to about 25 .

The second regime in Fig. 8 constitutes $\mathrm{SNR}_{\text {spatial }}$ scenes with moderate variability that have reduced contrast compared to the first regime, and these scenes may have relatively static scene contents that contribute to $\mathrm{SNR}_{T}$ only through the geolocation uncertainty mechanism. This contribution to $\mathrm{SNR}_{T}$ augments instrument noise but is no longer the primary source of variability that limits $\mathrm{SNR}_{T}$. The reasoning for this is that scene variability is not as efficient in entering the time domain as when the scene contains dynamic, high-contrast content. Thus, a unit increase in $\mathrm{SNR}_{\text {spatial }}$ threshold will not yield as much increase in $\mathrm{SNR}_{T}$. In this regime, changing the $\mathrm{SNR}_{\text {spatial }}$ threshold does not decrease instrument noise, and decreases in scene variability being manifested as temporal variability do not by themselves fully compensate for the change in $\mathrm{SNR}_{\text {spatial }}$. Thus, $\mathrm{dSNR}_{T} / \mathrm{dSNR}_{\text {spatial }}$ values of less than 1 are expected. In Fig. 8, this regime can be seen to extend from an $\mathrm{SNR}_{\text {spatial }}$ threshold of 25 to about 50 .

The third regime evident in Fig. 8 is represented by high SNR $_{\text {spatial }}$ scenes that are relatively homogeneous, and instrument noise is now considered to be a larger contributor to changes in scene $\mathrm{SNR}_{\text {spatial }}$ in time. In this regime though, increases in $\mathrm{SNR}_{T}$ become inevitable, based on the manner in which the $\mathrm{SNR}_{\text {spatial }}$ threshold is applied. The $\mathrm{SNR}_{\text {spatial }}$ threshold in this study is applied to all possible pixel clusters in each pair of consecutive GOES-R MESO images. This means that the threshold eliminates cases where instrument noise causes a significant decrease or increase in $\mathrm{SNR}_{\text {spatial }}$ in time. This will artificially constrain $\mathrm{SNR}_{T}$ to higher values.

An example illuminating this concept relates to rolling dice, as instrument noise is considered to be a random process in this study. After one roll of nine dice, all dice could have a value of 1. On the second roll, the dice values could vary between 1 and 6 . The reverse could also be true between the first and second rolls of the dice. This example is extreme, but such cases are expunged from this analysis. This would force smaller temporal variability in the population, which is reminiscent of the idea of "cherry-picking" data to get a better result. In this case, $\mathrm{SNR}_{T}$ is composed of instrument noise and a smaller contribution of $\mathrm{SNR}_{\text {spatial }}$ manifesting in the time domain. As $\mathrm{SNR}_{\text {spatial }}$ threshold increases, average $\mathrm{SNR}_{T}$ will have a component of increase based on artificially removing instrument noise. This reduction of instrument noise is expected to be less than the average $\mathrm{SNR}_{\text {spatial }}$ change, so $\mathrm{dSNR}_{T} / \mathrm{dSNR}_{\text {spatial }}$ values of less than 1 are again expected, but not necessarily the same as the second regime. In Fig. 8, the third regime extends from an $\mathrm{SNR}_{\text {spatial }}$ threshold of 50 to about 80. Note that, at about $\mathrm{SNR}_{\text {spatial }}$ threshold of 80 , there is a small uptick of $\mathrm{dSNR}_{T} / \mathrm{dSNR}_{\text {spatial }}$, but this could be due to relatively small sample size of around 1000 points.

When choosing a value of $\mathrm{SNR}_{\text {spatial }}$ threshold by which we estimate instrument low-light SNR, we attempt to strike a balance between eliminating worst offender and avoiding cherrypicking pixel clusters. If only the most homogeneous scenes are retained, we run the risk of overestimating instrument low-light SNR. Meanwhile, retaining high- to moderate-contrast inhomogeneous scenes will likely lead to underestimation of instrument low-light SNR. For this reason, we choose a baseline $\mathrm{SNR}_{\text {spatial }}$ threshold for ABI Ch 2 of 39.4, which lies in the middle of the second regime found in Fig. 8. This gives an $\mathrm{SNR}_{T}$ estimate of 57.1, as shown in Fig. 7. The error applied to this value is estimated by considering the range of $\mathrm{SNR}_{T}$ in the second regime of Fig. 8, as this is thought to be the region of most reliable data. When $\mathrm{SNR}_{\text {spatial }}$ threshold varies between 25 and $50, \mathrm{SNR}_{T}$ varies from about 49 to 65 . With respect to our estimate value of $\mathrm{SNR}_{T}$ of 57 , this gives an uncertainty of about \pm 8 . 
So, in attempting to determine the $\mathrm{ABI}$ Ch 2 low-light instrument SNR, there are unknowable sources of error in the analysis-e.g., how much does spatial variability manifested into temporal variability impact $\mathrm{SNR}_{T}$, and how much do we falsely inflate $\mathrm{SNR}_{T}$ as we try to increase $\mathrm{SNR}_{\text {spatial }}$ threshold beyond expected instrument SNR values? So the method in the case of ABI Ch 2 can be used to assert success or failure of the validation criteria, but the ability to measure the actual value of SNR can be limited by these unknowns. For other channels with less resolved bit depth, this can become a limiting factor as well.

\subsection{Advanced Baseline Imager Channel 1 and Channels 3 to 6 Low-Light Signal-to-Noise Ratio}

The other ABI VNIR bands-Chs 1 and 3 to 6-do not have explicit MRD low-light requirements, but it is still important to try to quantify their performance. For this reason, the low-light SNR method applied to ABI Ch 2 in the previous section is also applied to the remaining VNIR channels. In this section, the GOES-16 ABI Chs 1 and 3 to 6 low-light SNR results are summarized together. Note that the tables in this section also include results from ABI Ch 2, but refer to Sec. 3.1 for discussion.

For ABI VNIR Chs 1 and 3 to 6, Table 1 provides values for the following parameters that define the analysis population: estimated radiances at $2.5 \%$ and $7.5 \%$ albedo scenes, $L_{2.5 \%}$ and $L_{7.5 \%}$, for the solar subpoint; $\mathrm{SNR}_{\text {spatial }}$ threshold; and temporal radiance difference $(\Delta L)$ minimum and maximum, and histogram maximum number of samples, for pixels that pass the $\mathrm{SNR}_{\text {spatial }}$ threshold. Note that the values of $\mathrm{SNR}_{\text {spatial }}$ threshold were more difficult to assess from the analysis of $\mathrm{dSNR}_{T} / \mathrm{dSNR}_{\text {spatial }}$ in Chs 1 and 3 to 6 , because of much smaller population size. So, the analysis of other ABI channels in addition to $\mathrm{Ch} 2$ could be made more robust by longer data acquisitions.

In the mesoscale image depicted in Fig. 2, the distribution of low albedo scene regions that exceed the $\mathrm{SNR}_{\text {spatial }}$ threshold can vary between ABI Chs 1 and 3 to 6. For ABI Ch 1, a predominance of low albedo values lies over high-elevation land regions, e.g., over the Andes Mountains. The reason for this is that Rayleigh scattering at $0.47 \mu \mathrm{m}$ can give a topof-atmosphere measured albedo of over $20 \%$ over low-elevation targets. It is only over dark scenes and at very high elevation, where the amount of Rayleigh scattered light is much smaller, that this albedo can be lower. Similar to Ch 2 discussed in Sec. 3.1, Chs 3 and 5 are observed to have a predominance of low albedo values over ocean, but only Ch 3 has notable contiguous areas in these regions. For Ch 5, the low albedo values are quite scattered. Chs 4 and 6 have low albedos that are mainly exclusive to land areas. For $\mathrm{Ch} 4$, the reason for this is that strong water absorption of this band limits sensitivity to mainly cloud tops with extremely high altitude, such as towering cumulonimbus. Meanwhile, for $\mathrm{Ch} 6$, the reason that albedos within the threshold range were not found over oceans is that top-of-atmosphere albedo over ocean for this band can be quite low $(<2 \%)$.

In Figs. 9(a)-9(e), $\mathrm{SNR}_{T}$ versus mean albedo are shown for Chs 1 and 3 to 6 . The plots also include the computed mean and threshold of $\mathrm{SNR}_{\text {spatial }}$, as well as mean $\mathrm{SNR}_{Q}$. As shown in

Table 1 Parameters that define the viable low-light SNR analysis population.

\begin{tabular}{|c|c|c|c|c|c|}
\hline Ch \# & $\begin{array}{c}L_{2.5 \%} \\
\mathrm{~W} /\left(\mathrm{m}^{2} \mathrm{sr} \mu \mathrm{m}\right)\end{array}$ & $\begin{array}{c}L_{7.5 \%} \\
\mathrm{~W} /\left(\mathrm{m}^{2} \mathrm{sr} \mu \mathrm{m}\right)\end{array}$ & $\begin{array}{l}\mathrm{SNR}_{\text {spatial }} \\
\text { threshold }\end{array}$ & $\begin{array}{c}\text { Min/Max temporal } \Delta L \\
\left.\text { W/(m } \mathrm{m}^{2} \mathrm{sr} \mu \mathrm{m}\right)\end{array}$ & $\begin{array}{l}\text { Max number } \\
\text { of samples }\end{array}$ \\
\hline 1 & 16.05 & 48.16 & 10.0 & $-3 / 1$ & $<10^{2}$ \\
\hline 2 & 13.0 & 38.9 & 39.4 & $-3 / 3$ & $\sim 10^{6}$ \\
\hline 3 & 7.6 & 22.8 & 5.6 & $-2 / 2$ & $>10^{4}$ \\
\hline 4 & 2.9 & 8.6 & 16.4 & $-0.4 / 0.4$ & $\sim 10^{4}$ \\
\hline 5 & 1.9 & 5.8 & 8.2 & $-0.5 / 0.5$ & $>10^{2}$ \\
\hline 6 & 0.6 & 1.8 & 10.3 & $-0.15 / 0.15$ & $>10^{2}$ \\
\hline
\end{tabular}




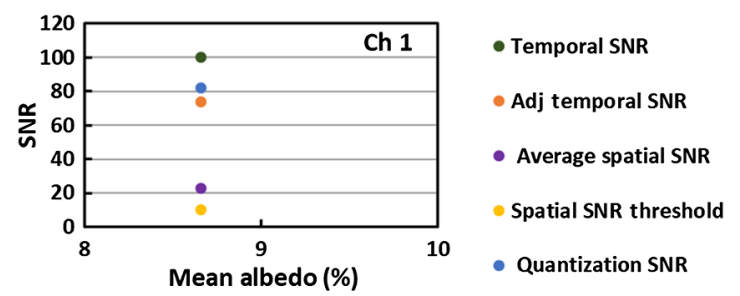

(a)

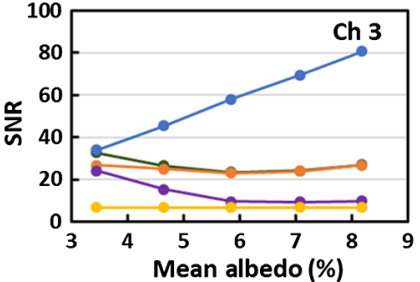

(b)

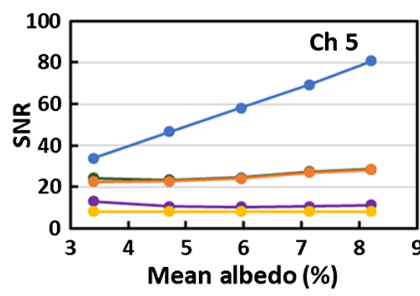

(d)

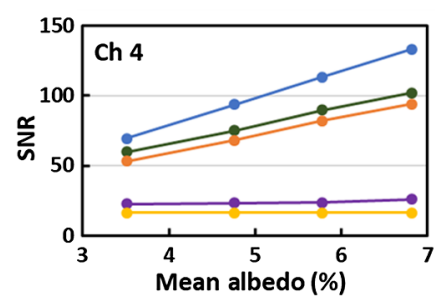

(c)

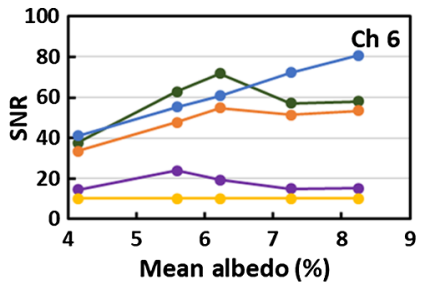

(e)

Fig. 9 For $\mathrm{ABI}$ (a) Ch 1, (b) Ch 3, (c) Ch 4, (d) Ch 5, and (e) Ch 6, the following parameters are depicted as a function of actual mean albedo: average and threshold of $\mathrm{SNR}_{\text {spatial }}, \mathrm{SNR}_{Q}$, and average $\mathrm{SNR}_{T}$ computed with and without substituting zero values of temporal radiance difference with the quantization radiance difference.

Figs. 9(a)-9(e), ABI L1b product quantization and the temporal radiance changes arising from meteorological evolution or INR instability over inhomogeneous scenes impact or limit the degree to which $\mathrm{SNR}_{T}$ can be determined. The effect of $\mathrm{SNR}_{\text {spatial }}$ as an inhibiting factor of $\mathrm{SNR}_{T}$ can be found to be most evident in Figs. 9(b)-9(e). In these cases, well-known INR instabilities present in the ABI L1b data product in this early stage of GOES-16 ABI validation could have been a contributor to translating spatial radiance variability into temporal radiance variability (personal communication-Vladimir Kondratovich). When albedo and $\mathrm{SNR}_{Q}$ are relatively small, $\mathrm{SNR}_{T}$ can be larger than $\mathrm{SNR}_{Q}$, and $\mathrm{SNR}_{T \text { adj }}$ is smaller than $\mathrm{SNR}_{Q}$. This is shown in Figs. 9(a), 9(b), and 9(e) and is due to the relatively small bit depth of 10 for these channels. In addition, when $\mathrm{SNR}_{Q}$ is larger, such as shown in Fig. 9(c), this has less of a bearing on limiting $\mathrm{SNR}_{T}$.

\section{Summary}

The GOES-R ABI has important MRD ${ }^{1}$ specifications related to instrument SNR for $100 \%$ albedo scenes. In addition, since the ABI AOD product depends on relatively low noise over low-light ocean scenes, there is also a requirement for ABI Ch 2 SNR for 5\% albedo scenes. The SNR value for the latter requirement was originally set to 50:1, which during ABI development needed to be waived to 20:1. For the purpose of comparing on-orbit ABI Ch 2 SNR with respect to prelaunch expected performance, a PLPT denoted as ABI-L1b-PLPT-009 was established. The success criteria for this test that relate most to low-light SNR is "Validating that low light SNR meets expected performance for ABI Band 2 at $5 \%$ reflectance by measurements or interpolation." In addition, for the purpose of this article, low-light SNR results for all other VNIR channels are shared as well. 
In this test, an analysis has been performed of ABI Mode-3 MESO 30-s images between 17:00 UTC and 17:15 UTC on May 23, 2017. The locations for the ABI MESO-1 and MESO-2 centers are identical at $8.975615^{\circ} \mathrm{S}$ latitude and $76.91671^{\circ} \mathrm{W}$ longitude. The data used for the analysis have been screened to significantly reduce the number of pixels with scene evolution associated with meteorology and image-to-image INR uncertainties in highly inhomogeneous scenes. The analysis is for low-light regions, which are isolated from the ABI data with radiance ranges defined by assuming $2.5 \%$ and $7.5 \%$ albedo scenes exposed to sunlight with a zero-degree SZA and an intensity based on a 1 AU Sun-Earth distance. The radiance range is divided into five radiance subintervals to ensure that actual scenes within a half a percent of $5 \%$ albedo are available to be analyzed.

In Table 2, the all-VNIR-channel average low-light radiance range subintervals have associated mean actual albedo values of $3.7 \%, 4.9 \%, 5.9 \%, 7.1 \%$, and $8.3 \%$, which encompass the targeted $5 \%$ albedo. The scene-to-scene $\mathrm{SNR}_{T}$, averaged over all six VNIR channels and five albedo values, is about $52 \pm 5$. This value drops to an adjusted $\mathrm{SNR}_{T}\left(\mathrm{SNR}_{T \text { adj }}\right)$ of about $48 \pm 5$ when pixels with $\Delta L[n]=0$ are substituted with root-two times the $L 1 \mathrm{~b}$ radiance scaling factor and a random sign. As stated in Sec. 3.1, subject-matter experts within GOES-R Program Systems Engineering established a prelaunch estimate of expected (baseline) postlaunch performance of ABI Ch 2 minimum and mean SNR at 5\% albedo of 44.2 and 64.5, respectively. In this analysis, the computed mean $\mathrm{SNR}_{T}$ and $\mathrm{SNR}_{T \text { adj }}$ validates this expectation with an estimated value of on-orbit ABI Ch 2 low-light SNR of $57 \pm 8$.

The main difficulty of this method is not being able to separate the temporal radiance measurement changes due to instrument noise from those caused by scene meteorological evolution and INR instability coupled with high scene spatial variability. To tackle this difficulty, the $\mathrm{SNR}_{\text {spatial }}$ threshold values are chosen with the aid of an analysis of the change of $\mathrm{SNR}_{T}$ with respect to change of average $\mathrm{SNR}_{\text {spatial }}\left(\mathrm{dSNR}_{T} / \mathrm{dSNR}_{\text {spatial }}\right)$ as a function of $\mathrm{SNR}_{\text {spatial }}$ threshold. An example shown for ABI Ch 2 illustrates how this analysis is used to help in choosing $\mathrm{SNR}_{\text {spatial }}$ threshold values that are a conservative compromise between removing worst offender spatial variability that produces erroneously low $\mathrm{SNR}_{T}$, and cherry-picking data that produce erroneously high $\mathrm{SNR}_{T}$. This analysis was also used to aid in producing an uncertainty estimate.

So this method in the case of $\mathrm{ABI}$ Ch 2 can be used to assert success or failure of the validation criteria, but the ability to measure the actual value of SNR can be limited by issues related to scene spatial variability and evolution. For other channels with less resolved bit depth, this can become a limiting factor as well. Finally, the large population size used to perform the ABI Ch 2 analysis was found to be essential.

Table 2 ABI VNIR channel SNR analysis results for the five low-light radiance range subintervals. Provided for each subinterval are albedo, temporal SNR $\left(\mathrm{SNR}_{T}\right)$, and adjusted temporal SNR $\left(\mathrm{SNR}_{\text {Tadj }}\right)$.

\begin{tabular}{|c|c|c|c|c|c|}
\hline Ch \# & $\begin{array}{c}\text { Albedo } \\
1 / \text { SNR }_{T} / \text { SNR }_{T \text { adj }}\end{array}$ & $\begin{array}{c}\text { Albedo } \\
2 / \mathrm{SNR}_{T} / \mathrm{SNR}_{\text {Tadj }}\end{array}$ & $\begin{array}{c}\text { Albedo } \\
3 / \mathrm{SNR}_{T} / \mathrm{SNR}_{\text {Tadj }}\end{array}$ & $\begin{array}{c}\text { Albedo } \\
4 / \mathrm{SNR}_{T} / \mathrm{SNR}_{T \mathrm{adj}}\end{array}$ & $\begin{array}{c}\text { Albedo } \\
\text { 5/SNR }_{T} / \text { SNR }_{\text {Tadj }}\end{array}$ \\
\hline 1 & No result & No result & No result & No result & $8.7 \% / 100 / 74$ \\
\hline 2 & $4.1 \% / 55 / 54$ & $4.9 \% / 57 / 57$ & $5.9 \% / 65 / 64$ & $7.2 \% / 75 / 74$ & $8.1 \% / 77 / 77$ \\
\hline 3 & $3.5 \% / 33 / 27$ & $4.6 \% / 26 / 25$ & $5.8 \% / 23 / 23$ & $7.1 \% / 24 / 24$ & $8.2 \% / 27 / 27$ \\
\hline 4 & $3.5 \% / 60 / 53$ & $4.6 \% / 76 / 68$ & $5.6 \% / 90 / 82$ & $6.6 \% / 102 / 94$ & No Result \\
\hline 5 & $3.4 \% / 24 / 22$ & $4.7 \% / 23 / 23$ & $6.0 \% / 24 / 24$ & $7.1 \% / 28 / 27$ & $8.2 \% / 28 / 28$ \\
\hline 6 & $4.1 \% / 38 / 34$ & $5.6 \% / 63 / 48$ & $6.2 \% / 72 / 55$ & $7.3 \% / 57 / 52$ & $8.3 \% / 58 / 53$ \\
\hline $\begin{array}{l}\text { All } \mathrm{Ch} \\
\text { average }\end{array}$ & $3.7 \% / 42 / 38$ & $4.9 \% / 49 / 44$ & $5.9 \% / 55 / 50$ & $7.1 \% / 57 / 54$ & $8.3 \% / 58 / 52$ \\
\hline
\end{tabular}




\section{Appendix A}

In this section, a more theoretical treatment is developed and discussed regarding the three $\mathrm{dSNR}_{T} / \mathrm{dSNR}_{\text {spatial }}$ regimes shown in Fig. 8. These three regimes are characterized, respectively, by an initial steep decline of $\mathrm{dSNR}_{T} / \mathrm{dSNR}_{\text {spatial }}$ with values $>1$, followed by two plateaus with values $<1$. The first plateau has a value $\mathrm{dSNR}_{T} / \mathrm{dSNR}_{\text {spatial }}$ of 0.9 , while the second plateau has an average value of about 0.75 . The theoretical development starts out with equations for $\mathrm{SNR}_{T}$ and $\mathrm{SNR}_{\text {spatial }}$, which can be alternatively be expressed as

$$
\mathrm{SNR}_{T}=\frac{\sqrt{2} R}{\left(2 \sigma_{I}^{2}+\sigma_{S \rightarrow T}^{2}\right)^{\frac{1}{2}}}
$$

and

$$
\mathrm{SNR}_{\text {Spatial }}=\frac{R}{\left(\sigma_{I}^{2}+\sigma_{S}^{2}\right)^{\frac{1}{2}}} .
$$

In these equations, $R$ is the average scene radiance, while $\sigma_{I}, \sigma_{S}$, and $\sigma_{S \rightarrow T}$ are the standard deviations associated with instrument noise, spatial variability, and spatial variability manifested into temporal variability. The latter mechanism primarily occurs over 30 -s time periods due to meteorological evolution in a scene and/or geolocation uncertainty in an inhomogeneous scene. So the resultant noise in $\mathrm{SNR}_{T}$ is due to a combination of instrument noise and scene variability that manifests into the time domain, while $\mathrm{SNR}_{\text {spatial }}$ depends on a combination of instrument noise and scene variability.

The focus here is on how $\sigma_{S}$ and $\sigma_{S \rightarrow T}$ change with $\mathrm{SNR}_{\text {spatial }}$ threshold is reflected in $\mathrm{dSNR}_{T} / \mathrm{dSNR}_{\text {spatial }}$ changes. This can be found by finding the ratio of the derivatives of $\mathrm{SNR}_{T}$ and $\mathrm{SNR}_{\text {spatial }}$, which can be expressed as

$$
\begin{gathered}
\frac{\mathrm{dSNR}_{T}}{\mathrm{dSNR}_{T}(\text { Thresh })}=-\frac{\sqrt{2} R \sigma_{S \rightarrow T}}{\left(\left(2 \sigma_{I}^{2}+\sigma_{S \rightarrow T}^{2}\right)^{3}\right)^{\frac{1}{2}}} \frac{\mathrm{d} \sigma_{S \rightarrow T}}{\mathrm{dSNR}_{T}(\text { Thresh })}, \\
\frac{\mathrm{dSNR}_{\text {spatial }}}{\mathrm{dSNR}_{T}(\text { Thresh })}=-\frac{R \sigma_{S}}{\left(\left(\sigma_{I}^{2}+\sigma_{S}^{2}\right)^{3}\right)^{\frac{1}{2}}} \frac{\mathrm{d} \sigma_{S}}{\mathrm{dSNR}_{T}(\text { Thresh })},
\end{gathered}
$$

and

$$
\frac{\mathrm{dSNR}_{T}}{\mathrm{dSNR}_{\text {spatial }}}=\frac{\sqrt{2} \sigma_{S \rightarrow T}\left(\left(\sigma_{I}^{2}+\sigma_{S}^{2}\right)^{3}\right)^{\frac{1}{2}}}{\sigma_{S}\left(\left(2 \sigma_{I}^{2}+\sigma_{S \rightarrow T}^{2}\right)^{3}\right)^{\frac{1}{2}}} \frac{\mathrm{d} \sigma_{S \rightarrow T}}{\mathrm{~d} \sigma_{S}} .
$$

Note that the average radiance associated with $5 \%$ albedo is held constant for both $\mathrm{dSNR}_{T}$ and $\mathrm{dSNR}_{\text {spatial }}$, and so $\mathrm{d} R$ is not relevant to this analysis. To exemplify the regimes that we might see according to the $\mathrm{dSNR}_{T} / \mathrm{dSNR}_{\text {spatial }}$ parameter, we assume scene variability itself is on average larger than the amount of scene variability that manifests into the time domain until scene variability is small compared to instrument noise. This will be discussed further later. Below, we give four examples of how the $\mathrm{dSNR}_{T} / \mathrm{dSNR}_{\text {spatial }}$ parameter changes, depending on the relative magnitudes of $\sigma_{I}$ with respect to $\sigma_{S}$ and $\sigma_{S \rightarrow T}$. These four examples encompass when $\sigma_{I}$ is much smaller than, slightly less than, slight greater than, and much greater than $\sigma_{S}$ and $\sigma_{S \rightarrow T}$. These examples are given as follows.

1. If $\sigma_{I}$ is much smaller than $\sigma_{S}$ and $\sigma_{S \rightarrow T}-\mathrm{e}$.g., assume $\sigma_{S}=5 \sigma_{I}$ and $\sigma_{S \rightarrow T}=2.5 \sigma_{I}$

$$
\frac{\mathrm{dSNR}_{T}}{\mathrm{dSNR}_{\text {spatial }}}=\frac{\sqrt{2} \sigma_{I}\left(\left(\sigma_{I}^{2}+25 \sigma_{I}^{2}\right)^{3}\right)^{\frac{1}{2}}}{2 \sigma_{I}\left(\left(2 \sigma_{I}^{2}+6.25 \sigma_{I}^{2}\right)^{3}\right)^{\frac{1}{2}}} \frac{\mathrm{d} \sigma_{S \rightarrow T}}{\mathrm{~d} \sigma_{S}} .
$$

When $\mathrm{SNR}_{\text {spatial }}$ threshold is first increased away from zero, most (if not all) of the data to be removed have large spatial variation due to scene inhomogeneity. The spatial and 
temporal variations of these pairs are not necessarily intimately coupled. For example, if a high gradient area moves into a scene pixel over the 30-s interval, the radiance difference can be much larger than the spatial variation. If a pixel has high spatial variation due to coastline or stationary cloud edge, the radiance difference can be zero. It is expected that $\mathrm{d} \sigma_{S \rightarrow T} / \mathrm{d} \sigma_{S}$ will be around one or greater, as the worst cases of scene variability manifesting into the time domain are eliminated, even for small changes of $\sigma_{S}$. In addition, the term operating on $\mathrm{d} \sigma_{S \rightarrow T} / \mathrm{d} \sigma_{S}$ would be on the order of 4 . So $\mathrm{dSNR}_{T} / \mathrm{dSNR}_{\text {spatial }}$ would be expected to be $>1$.

2. If $\sigma_{I}$ is slightly smaller than $\sigma_{S}$ and $\sigma_{S \rightarrow T}$-e.g., assume $\sigma_{S}=2 \sigma_{I}$ and $\sigma_{\mathrm{S} \rightarrow \mathrm{T}}=\sigma_{I}$

$$
\frac{\mathrm{dSNR}_{T}}{\mathrm{dSNR}_{\text {spatial }}}=\frac{\sqrt{2} \sigma_{I}\left(\left(\sigma_{I}^{2}+2 \sigma_{I}^{2}\right)^{3}\right)^{\frac{1}{2}}}{2 \sigma_{I}\left(\left(2 \sigma_{I}^{2}+\sigma_{I}^{2}\right)^{3}\right)^{\frac{1}{2}}} \frac{\mathrm{d} \sigma_{S \rightarrow T}}{\mathrm{~d} \sigma_{S}} .
$$

It is expected that the change of $\mathrm{d} \sigma_{S \rightarrow T} / \mathrm{d} \sigma_{S}$ will be slightly greater than or about one. This could be a regime where scenes with moderate homogeneity do not evolve in time and geolocation variability is the primary cause of temporal variability. The term operating on it would be on the order of $1 / \sqrt{2}$, so $\mathrm{dSNR}_{T} / \mathrm{dSNR}_{\text {Spatial }}$ would be about 1 or slightly less.

3. If $\sigma_{I}$ is slightly larger than $\sigma_{S}$ and $\sigma_{S \rightarrow T}$-e.g., assume $\sigma_{S}=\sigma_{I}$ and $\sigma_{S \rightarrow T}=\sigma_{I} / 2$

$$
\frac{\mathrm{dSNR}_{T}}{\mathrm{dSNR}_{\text {spatial }}}=\frac{\sqrt{2} \sigma_{I}\left(\left(\sigma_{I}^{2}+\sigma_{I}^{2}\right)^{3}\right)^{\frac{1}{2}}}{2 \sigma_{I}\left(\left(2 \sigma_{I}^{2}+0.25 \sigma_{I}^{2}\right)^{3}\right)^{\frac{1}{2}}} \frac{\mathrm{d} \sigma_{S \rightarrow T}}{\mathrm{~d} \sigma_{S}} .
$$

In this case, the change of $\mathrm{d} \sigma_{S \rightarrow T} / \mathrm{d} \sigma_{S}$ will be about one, as both spatial and temporal variability become increasingly related. There are two reasons for this behavior. The first is that whenever a scene has spatial variability, instrument geolocation uncertainty will transform a portion of this spatial variability into temporal variability. The second is the nature of instrument noise, which is assumed to be a random occurrence equally in space and time. Thus, pixel radiance variability with respect to its neighbors in a scene and pixel variability from one 30-s image to another are tied to the same noise process. As instrument noise begins to dominate compared to scene feature variability, further removal of pixels by increasing $\mathrm{SNR}_{\text {spatial }}$ threshold will have a statistically proportional impact on the spatial and temporal SNR of the remaining pixels. This we call cherrypicking the data, which we want to avoid. Meanwhile, the term operating on $\mathrm{d} \sigma_{S \rightarrow T} / \mathrm{d} \sigma_{S}$ would be on the order of $3 / 5$. So $\mathrm{dSNR}_{T} / \mathrm{dSNR}_{\text {Spatial }}$ would be about $3 / 5$.

4. If $\sigma_{I}$ is much larger than $\sigma_{S}$ and $\sigma_{S \rightarrow T}-$ e.g., assume $\sigma_{S}=\sigma_{I} / 5$ and $\sigma_{S \rightarrow T}=5$

$$
\frac{\mathrm{dSNR}_{T}}{\mathrm{dSNR}_{\text {spatial }}}=\frac{\sqrt{2} \sigma_{I}\left(\left(\sigma_{I}^{2}+0.04 \sigma_{I}^{2}\right)^{3}\right)^{\frac{1}{2}}}{\sigma_{I}\left(\left(2 \sigma_{I}^{2}+0.04 \sigma_{I}^{2}\right)^{3}\right)^{\frac{1}{2}}} \frac{\mathrm{d} \sigma_{S \rightarrow T}}{\mathrm{~d} \sigma_{S}} .
$$

Based on the discussion for example 3, the change of $\mathrm{d} \sigma_{S \rightarrow T} / \mathrm{d} \sigma_{S}$ is expected to be about one, as changes in $\sigma_{S}$ and $\sigma_{S \rightarrow T}$ are predominantly tied to the same instrument noise process. The term operating on $\mathrm{d} \sigma_{S \rightarrow T} / \mathrm{d} \sigma_{S}$ would be on the order of $1 / 2$, so $\mathrm{dSNR}_{T} / \mathrm{dSNR}_{\text {Spatial }}$ would be expected to be around $1 / 2$.

From this analysis, regimes 1 to 3 in Fig. 8 could be explained mainly by examples 1 to 3 above, but there are gray areas. Since the peak value is just over 3 in Fig. 8, which is attributed to regime 1 , it is possible that these scenes are only moderately inhomogeneous. This would be consistent for low-light ocean scenes in the middle of the day. Also regime 3 could include pixels that are featureless and are dominated by instrument noise, so regime 3 could be a hybrid of examples 3 and 4. Such gray areas are an artifact of taking derivatives of statistically determined variables. 


\section{Acknowledgments}

The author would like to acknowledge that this work was fully supported by GOES-R Program funding. They would also like to thank all STAR Calibration Working Group colleagues for their input during the process of writing this article.

\section{Disclaimer}

The article's contents are solely the opinions of the author(s) and do not constitute a statement of policy, decision, or position on behalf of NOAA or the U.S. Government.

\section{References}

1. "GOES-R Mission Requirements Document," 410-R-MRD-0070, V3.28, 122 p. (2019).

2. "GOES-R Advanced Baseline Imager (ABI) algorithm theoretical basis document for suspended matter, aerosol optical depth, and aerosol size parameter," NOAA/NESDIS/STAR, Version 4.2, 112 p., 2018, https:/www.star.nesdis.noaa.gov/goesr/documents/ATBDs/ Baseline/ATBD_GOES-R_Aerosol_Optical_Depth_v4.2_Feb2018.pdf (accessed February 17, 2020).

3. "Geostationary Operational Environmental Satellite (GOES)—R series ABI beta, provisional and full validation readiness, implementation and management plan (RIMP)," Version 1.1, 416-R-RIMP-0315, 50 pp. (Document available upon request from GOES-R Ground Segment Product Readiness and Operations (PRO) Team Lead Matthew. Seybold@noaa.gov) (2017).

4. S. Kalluri et al., "From photons to pixels: processing data from the Advanced Baseline Imager," Remote Sens. 10(2), 177 (2018).

5. T. J. Schmit et al., "A closer look at the ABI on the GOES-R series," Bull. Am. Meteorol. Soc. 98, 681-698 (2017).

6. "GOES R series product definition and users' guide (PUG): Volume 3: level 1b products," 416-R-PUG-L1B-0347 Version 2.2, 402 p., 2019, https://www.goes-r.gov/users/docs/PUGL1b-vol3.pdf. (accessed February 17, 2020).

Robbie Iacovazzi is a calibration engineer at NOAA/NESDIS Center for Satellite Applications and Research. For nearly two-decades he has performed instrument and product integrity cal/val activities for space-based microwave sounding and VNIR imaging radiometers manifested on NOAA operational and NASA research satellites.

Xiangqian Wu leads calibration support for NOAA's operations of Advanced Very High Resolution Radiometer (AVHRR) on POES (since 2002), Imager and Sounder on GOES (since 2004), Ozone Mapper Profiler Suite (OMPS) on S-NPP (2011-2014), and Advanced Baseline Imager (ABI) on GOES-R (since 2014). He has been a member of the WMO-sponsored Global Space-based Inter-Calibration System (GSICS) Research Working Group since its inception in 2006, and served as its first chair. 